\title{
Vision-Based Method for Forward Vehicle Brake Lights Recognition
}

\author{
${ }^{1}$ Wei Liu, ${ }^{1,2}$ Hong Bao*, ${ }^{3}$ Jun Zhang and ${ }^{2}$ Cheng Xu \\ ${ }^{1}$ Information College of Beijing Union University, 100101, Beijing, China \\ ${ }^{2}$ Beijing Key Laboratory of Information Service Engineering, 100101, Beijing, \\ China \\ ${ }^{3}$ Management Sciences College of Chengdu University of Technology, 610052, \\ Chengdu, China \\ 'sophia_hello@163.com
}

\begin{abstract}
This paper presents a recognition algorithm combining the vehicle detection and the color difference of $R G B$ color space to recognize the brake-lights state of moving vehicles in order to achieve the intelligent-car's rear-end collision warning about the vehicle in front of it. Firstly, we train and build AdaBoost cascade classifier by haar features samples and scaling sub-windows are used to detect the target vehicle from the region of interest of the resized image. Then we compare the adjacent frames to recognize brake light status, which including using color, shape, structural features to identify the third brake light; comparing the center of gravity coordinates and the color difference threshold to rear brake lights when vehicles are not red or yellow; according to subtraction of each RGB corresponding channel, binarization, and the color difference threshold of RGB color space to identify the red or yellow vehicles' brake lights. Finally, experiments show that the algorithm can detect the front vehicle's braking quickly and accurately.
\end{abstract}

Keywords: vehicle detection, brake lights recognition, regions of interest, classifier

\section{Introduction}

Recently, with a lot of research work being carried out on intelligent driving, safety of autonomous vehicles issues is becoming more and more striking. As intelligent cars can travel in complex environment and no interference, therefore, taking security issues of intelligent driving into account, detection and identification of the vehicle braking state (rear brake light state) of front cars is necessary. With the advantages of low cost cameras and other equipment, it becomes more and more widely used by image processing method based on visual detection of the front of the event state (for example, the front of the vehicle braking state). This application can warn drivers or the autopilot system to take appropriate action to avoid potential collision conflicts.

Autonomous vehicles can take appropriate measures (such as normal driving, deceleration, braking) by implications of taillight states so as to further ensure the safety of vehicles.

Therefore, it makes great sense that proposing a low-cost, effective brake lights state identification method of front vehicle to accommodate future autopilot market, so that the vehicle can travel on the road safely and reliably. It has strong social and practical significance. 


\section{Related Works}

In related research work, Wei-Yen Wang [1] used digital electronic circuitry to detect vehicle' taillights before their vehicle at night.

By using the red channel signal from the CCD camera as the input signal of the voltage comparator and comparing with threshold, the output of the comparator will produce a horizontal scanning line of two positive pulses in order to detect and recognize the vehicle taillight. This method can detect vehicle taillights in non-contact and non-reflective way, but in the case of multiple sets of tail lights, single or bad taillight, blurred taillights, this algorithm does not conduct experiments, and the algorithm is applied to the car taillights detection at night.

Using digital image processing method to detect and recognize traffic lights [2-6], tail lights to detect the front vehicles [7-18], detect headlights [19], detect headlights and taillights[20], and brake lights [21-25].In [21], the method differs from the literature [220] in the spatial domain for processing. Literature [21] detects in the frequency domain brake lights at night, and compares with the previous frame pictures brake lights are turned off. When the brake lights, the picture will appear some obvious bright areas. Since the brake lights forming regions are sharply different from the surrounding environment at night, by the time-frequency domain transform can detect the peak frequency amplitude curve, so as the brake lights are detected. This method is suitable for the detection of the evening, but due to the interference of light in the day, it cannot get ideal results, and it has the computational complexity, there are some limitations. Literature [22-24] are based on Nakagami-m distribution of car taillights brake signal analysis, and models by finding the invariant features of the scattering characteristics of the brake lights signal to detect brake lights. This method is suitable for detection similarly in the evening, also susceptible to lights, and the model is more complex. Literature [25], using a Kalman filter to track the taillight area of front vehicle, uses the left and right taillight area mean brightness trends (curves) to determine the status of the lamp to detect the turn signal and brake lights of the vehicle. This method does not consider the red vehicles and broken brake lights situations, so there are some limitations.

Most of the literature of the vehicle brake lights state identification methods are mainly detection and identification of the vehicle's brake lights at night. Adopting these methods to detect brake lights has some accuracy, but affected by sample characteristics, these methods are suitable for detecting brake lights at night, and not suited to the detection of vehicles during the day. Because in the daytime, vehicle brake lights areas are compared with the surrounding environment of vehicle, the brightness is not very different. And this method is greatly affected by the light. Daytime scene is complex and difficult to detect, and the frequency domain and Nakagami-m distributed computing model are also complicated.

To solve those existing problems that vehicles brake lights are not suitable for detecting at daytime and models calculation is complex, this paper proposes a method to identify the state of the vehicle brake lights. By extracting color, shape, structure and other characteristics of the brake lights in the region and using the color difference threshold of RGB color space, we can recognize the brake-lights state of moving vehicles quickly and accurately, so as to ensure the safety of auxiliary driving and automatic driving.

\section{The Proposed Method}

\subsection{Vehicle Detection}

3.1.1. AdaBoost Algorithm: AdaBoost (Adaptive Boosting) algorithm which is an adaptive algorithm proposed by Freund \& Schapire [26], is an iterative algorithm. For a 
set of training set, firstly, obtain different training set Si by changing the probability distribution of each sample, then training each $\mathrm{Si}$ to obtain a weak classifier hi, and lastly combine these weak classifiers according to the different weights to get the strong classifier, meanwhile, for the weights of each classification, the higher accuracy of its classification, the higher of its weights. After repeated iterative training several times, the classification error approach to zero.

As is described in the following procedure:

(1)Normalize weight

$$
\mathrm{q}_{\mathrm{t}, \mathrm{i}}=\frac{w_{t, i}}{\sum_{j=1}^{n} w_{t, i}}
$$

Where $\mathrm{q}_{\mathrm{t}, \mathrm{i}}$ is the weight of the T iteration of sample ${ }^{x_{i}}$.

(2)For each feature $f$, training a weak classifier hi, then calculating the weighted error rate $\xi_{\mathrm{f}}$ of all the characteristics.

$$
\mathrm{h}\left(x_{i}, f, p, \theta\right)= \begin{cases}1 & p f\left(x_{i}\right)<p \theta \\ 0 & \text { else }\end{cases}
$$

Where a weak classifier $\mathrm{h}\left(x_{i}, f, p, \theta\right)$ composed of Sub-window image ${ }^{x_{i}}$, a feature $\mathrm{f}, \mathrm{p}$ that indicates the direction of inequality and threshold value $\theta$.

$$
\xi_{\mathrm{f}}=\Sigma_{i} q_{i}\left|h\left(x_{i}, f, p, \theta\right)-y_{i}\right|
$$

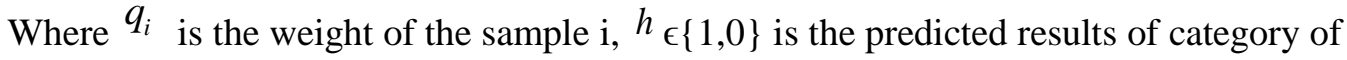
the sample $\mathrm{i},{ }^{y_{i}} \in\{1,0\}$ is the true category label of the sample $\mathrm{i}$.

(3) Select the weak classifier hi which has the minimum error rate $\xi_{\mathrm{f}}$.

(4) Adjust the weight

$$
w_{t+1, i}=w_{t, i} \beta_{t}^{1-e_{i}}
$$

Where ei $=0$ means that $\mathrm{xi}$ is correctly classified, ei $=1$ means that $\mathrm{xi}$ is wrongly classified; ${ }{ }_{t}=\frac{\xi_{\mathrm{t}}}{1-\xi_{t}}$.

(5) Combination of $\mathrm{T}$ optimal weak classifiers to get strong classifier

$$
C(\mathrm{x})= \begin{cases}1 & \sum_{t=1}^{T} \alpha_{t} h_{t}(x) \geq \frac{1}{2} \sum_{t=1}^{T} \alpha_{t} \\ 0 & \text { else }\end{cases}
$$

Where $\alpha_{\mathrm{t}=} \log \frac{1}{\beta_{\mathrm{t}}}$

3.1.2. Classifier Training: The object detection has been initially proposed by Paul Viola [Viola01] and improved by Rainer Lienhart [Lienhart02].First of all, using samples of haar feature to train classifier, we can get a cascade Adaboost classifier.

Training samples are divided into positive samples and negative samples. Positive samples which refer to the target samples to be tested, contains only the samples of the rear close-up images; Negative samples that do not contain the target of another images, 
such as the images containing the roads, traffic signs, buildings, billboards, cars, motorcycles, tricycles, pedestrians and bicycles, etc. All positive sample images have been normalized to uniform size.

Figure 1 is a cluster of training samples, among them positive training samples require the rear close-up images, but vastly different car rear shape, So the training sample selection process should take into the diversity of the samples account. Negative training samples are representative and each sample is not identical.

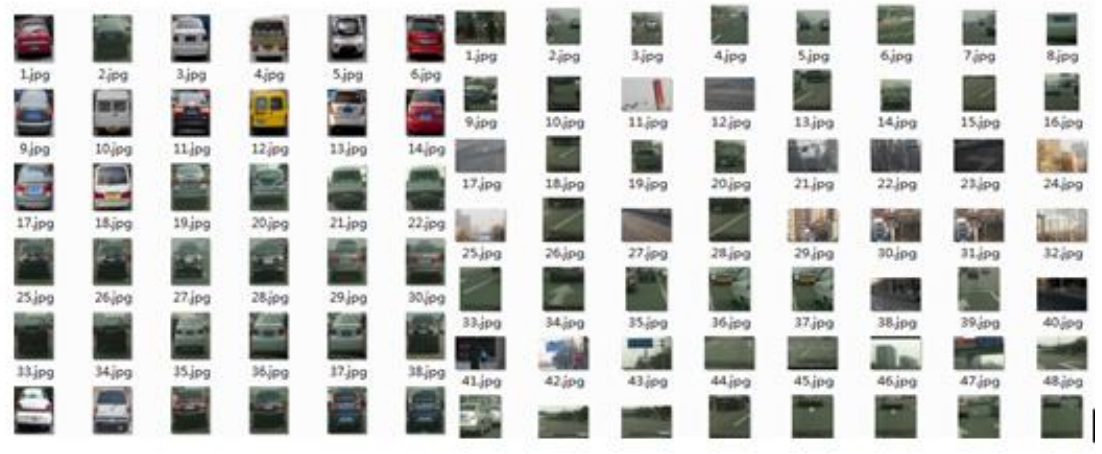

\section{Figure 1. Part of the Training Set of Positive Samples and Negative Samples}

The training process is divided into three steps: first of all, we need to extract Haar features; then we convert them to the corresponding Haar feature weak classifiers; finally optimal weak classifier is selected iteratively from a large number of weak classifiers.

\section{A. Extract Haar Features}

Haar-like feature is a simple rectangle feature introduced by Viola et al. [27-28] in its Face Detection System, because Haar-like wavelet (Haar wavelet) is named, which is defined as the difference of the sum of the pixel gray with area adjacent in image, this rectangle feature can reflect the gray change of the detection object local feature,due to the application of thought integral image to calculate Haar-like wavelet feature, which greatly improved the speed of getting the characteristics for the detector. Lienhart [29] et al. proposed extension of the Haar-like feature on the basis of the algorithm by Viola. The algorithm not only use the horizontal and vertical direction which is a rectangular region as the feature, but also rotate the rectangle to obtain the rectangle features of 45 degree angle, and they proposed a method for fast calculation of feature of 45 degree, such that the recognition performance of the system is significantly improved and the speed is not severely affected.

In this paper, we use horizontal and vertical rectangular region as the features.

\section{B. Generate Weak Classifier}

Each feature of Haar corresponds to a weak classifier and every weak classifier is defined by its characteristic parameters. Accounting the training samples, according to the above characteristics location information of Haar, to get the corresponding characteristic parameters. The weak classifier trained in AdaBoost algorithm in is any classifier, including the decision tree, neural network and hidden markov models and if the weak classifier is linear neural network, AdaBoost algorithm will construct a multi-layer perceptron node every time. 


\section{Use AdaBoost Algorithm to Select the Optimal Weak Classifier}

The training process of AdaBoost algorithm is to select the optimal weak classifier, and endow weight, Figure 2 is the sketch map of the training of AdaBoost algorithm.

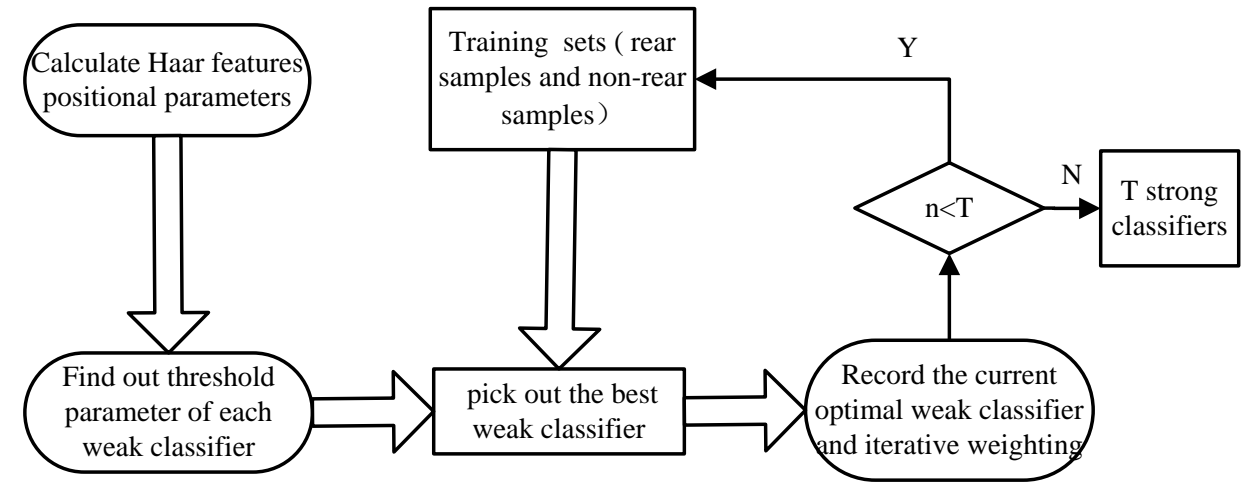

Figure 2. The Training Flowchart of AdaBoost Algorithm

\subsubsection{Object Detection}

Firstly, we resize each original image to the size $(352 * 288)$ and set the region of interest (ROI) for the resized image according to the prior knowledge, denoted for ROI1, in which we detect the target vehicle. Then scaling sub-windows are used to detect the target vehicle from ROI of the resized image. By using the Adaboost cascade classifier of rear close-up image sample training, we can find the rectangle area containing the object in the image, and the areas will be returned as a sequence of rectangular box. With the region's traversal search, we can get accurate area information of forward vehicle in ROI1. Finally, we reset ROI for the detected vehicle area and it is denoted for image Pic.

\subsection{Brake Lights Recognition}

We capture video on the road from Beijing Airport Terminal Buildings to central beijing by locating a monocular digital camera above the car windscreen. The size of images is $1280 * 720$. The frame rate is $30 \mathrm{fps}$. The maximum vehicle speed is limited to $80 \mathrm{~km} / \mathrm{h}$. Through the analysis of the relations among vehicle speed, braking distance and size of car; it indicates that the braking distance is about $20 \mathrm{~m}$ when the maximum speed is $80 \mathrm{~km} / \mathrm{h}$. In this paper, the minimum size of detection window is $50^{*} 60$ and the programming environment is vs2010+opencv2.4.4. The screen shot of recognition system is shown in Figure 3.

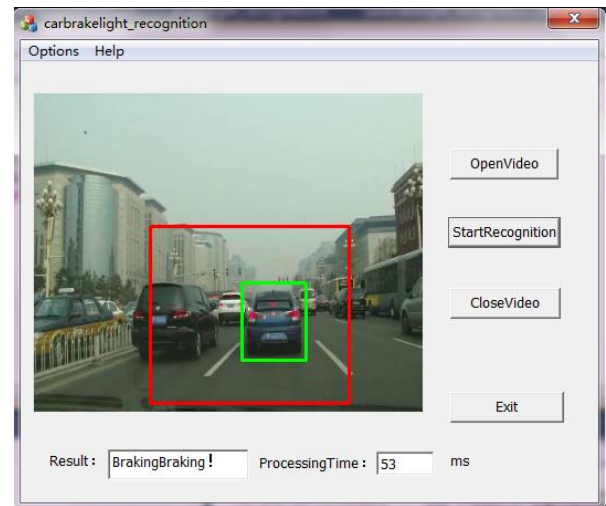

Figure 3. Brake Lights Recognition System Diagram 
Some samples of detected cars which are braking is shown in Figure 4.The average values of R,G,B channel of 300 red, 300 white and 300 yellow brake lights region samples are analyzed to get the red feature vector $\mathrm{Mr}=(\mathrm{Xi})$, the white feature vector $\mathrm{Mw}=(\mathrm{Yi})$ and the yellow feature vector $\mathrm{My}=(\mathrm{Zi}), \mathrm{i}=1,2,3$. Image frames are extracted from every two frames. The previous image including vehicle region is denoted as Pic1 and the next frame is denoted as Pic2. The basic braking recognition diagram is shown in Figure 5.
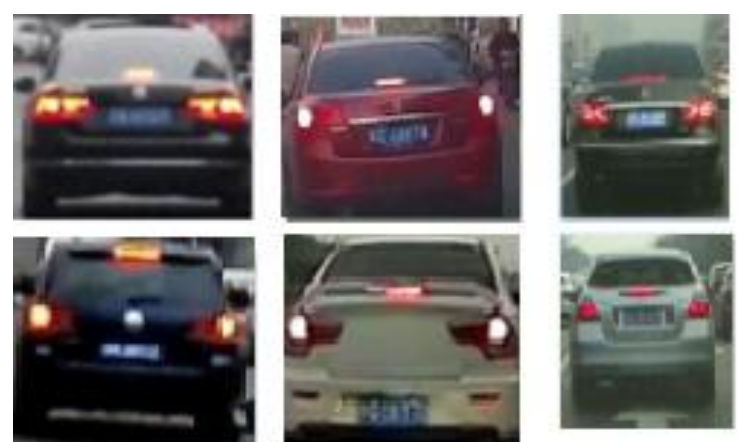

Figure 4. Some Samples of Detected Cars which are Braking

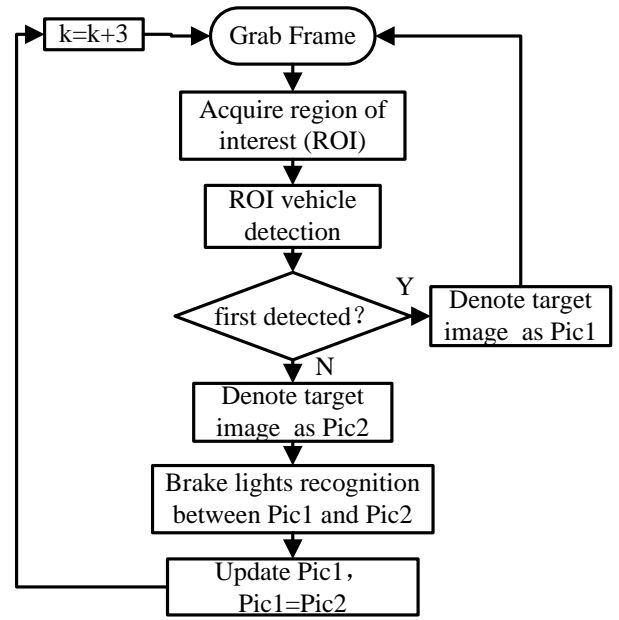

Figure 5. Overview of the Proposed Approach

\subsubsection{The Image Similarity Detection between the Two Frames}

This paper uses EMD to judge the similarity of two images by resizing the Pic1 and Pic2 to the same size $\left(a^{*} b\right)$.The smaller of the results (0) value represents that the matching degree is higher, it means that the vehicle in the two picture is the same car. In this case, the algorithms go to step 3.2.2 to identify the brake light, otherwise it outputs inserting road warning, go to step 4.

The OpenCv provides 5 kinds of contrast histogram mode: CORREL, CHISQR, INTERSECT, BHATTACHARYYA, EMD, where CHISQR is the fastest, EMD is the slowest and has many limitations, but EMD can get the best effect.

EMD is a kind of measurement criteria, what it is actually measured is how to transform a histogram for another histogram, including transform all or part of histogram to a new position, this kind of measurement can be done in any dimension histogram. Light can cause drift of image color values, although these drift does not change color histogram, but the drift makes the color value position change, resulting in some histogram matching strategy failure. If we use the histogram distance measuring instead 
of color histogram matching strategy, then we can still compare the distance of two histogram image just as histogram comparison, even if the second histogram drift occurs, we also can find the smallest distance metric.

3.2.2. Non-red and Non-yellow Car Brake Lights Recognition: In the paper, $t 1=1$ means that the third brake light is detected in the previous frame image, $\mathrm{t} 2=1$ means that the third brake light is detected in the next frame image.

By making subtraction between $R$ channel and $G$ channel in color space for Picture Pic1 and Picture Pic2, binarization and calculation of the proportion of white of binarized images, we can finally get $\mathrm{k} 1, \mathrm{k} 2$ respectively, if $\mathrm{k} 1<0.2 \& \& \mathrm{k} 2<0.2$, go to step A, otherwise go to step 3.2.3.

\section{A. The Third Brake Light Recognition}

Searching the contour of the two binary image of the upper part in the two frame images respectively, detecting the third brake light, features mainly including color, shape, structure. By finding contours, if the contour features meet the rectangular outline conditions and in the vicinity of the center of the axis, then the third brake light is detected.As is shown in Figure 6, (a), (b) two frame images to be compared, (c), (d) binarized image in the upper portion of the two frame images.

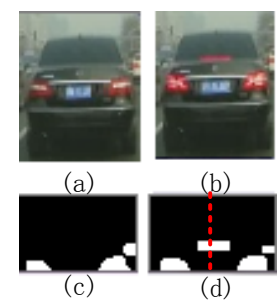

Figure 6. The Third Brake Light Detection Schematic Diagram

When $\mathrm{t} 1=0 \& \& \mathrm{t} 2=1$ or $\mathrm{t} 1=1 \& \& \mathrm{t} 2=1$, the brake state of frontal vehicle is detected, go to step 4 . When $\mathrm{t} 1=0 \& \& \mathrm{t} 2=0$, go to step B.

\section{B. Rear Brake Light Recognition}

Searching the contour of the two binary image of the lower part in the two frame images respectively to find two most closed coordinates of the center of gravity of the two contour sequences between two frame images, it is determined as follows:

$P=(x i, y i), Q=(x j, y j), i=1,2 \ldots k \ldots n 1, j=1,2 \ldots 1 \ldots n 2, n 1, n 2$, Where $P=(x i, y i)$ and $\mathrm{Q}=(\mathrm{xj}, \mathrm{yj})$ are two image contours sequences.

Min $\{|\mathrm{P}, \mathrm{Q}|\}$ stands for minimum distance, (xk, yk) and (xl, yl) corresponding to the coordinates of the center of gravity of the contour respectively.

As is shown in Figure 7, (a), (b) two frame images to be compared, (c), (d)binarized image in the lower portion of the two frame images. (e) extraction of the rectangular region of the rear brake light. 


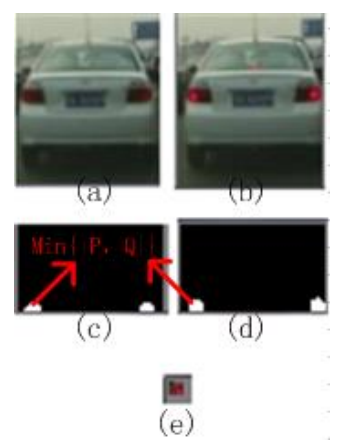

Figure 7. The Rear Brake Light Extraction Schematic Diagram

We take the two most closed coordinates of the center of gravity as the center, extract of the $5 \times 5$ rectangular region of two original images, then obtain feature vector $\mathrm{M} 1=$ $(\mathrm{Ai}), \mathrm{M} 2=(\mathrm{Bi}), \mathrm{i}=1,2,3$, and calculate the color difference $|\mathrm{M} 1, \mathrm{M} 2|$.The color difference of two colors is subtraction of the maximum value of positive and the minimum value of negative of the difference between the two colors corresponding channel levels. If there is no positive or negative, the maximum value of positive or negative equals to 0 .

If it meets the threshold conditions, i.e., $|\mathrm{M} 1, \mathrm{M} 2|<\mathrm{a}$, it indicates that the two frames have small differences. we obtain feature vector $\mathrm{M} 2$, compared with red vector $\mathrm{Mr}$ $=(\mathrm{Xi})$, white feature vector $\mathrm{Mw}=(\mathrm{Yi})$, and yellow feature vector $\mathrm{My}=(\mathrm{Zi})$ respectively, $\mathrm{i}=1,2,3$, for color difference threshold determination, in order to further confirm whether there is the rear brake light or not, determination methods are as follows:

If it meets the threshold conditions, i.e., $(|\mathrm{M} 2, \mathrm{Mr}|<=\mathrm{b})$ or $(|\mathrm{M} 2, \mathrm{Mw}|<=\mathrm{b})$ or $(\mid$ $\mathrm{M} 2, \mathrm{My} \mid<=\mathrm{b}),|\mathrm{M} 2, \mathrm{Mr}|$ represents the color difference of two vectors, it indicates that the frontal vehicle is braking, go to step 4 . Where a, b is statistical error threshold of color difference.

If it does not meet the threshold conditions, it indicates that the two frames have big differences. we obtain feature vector $\mathrm{M} 1$ and $\mathrm{M} 2$,compared with red vector $\mathrm{Mr}=(\mathrm{Xi})$, white feature vector $\mathrm{Mw}=(\mathrm{Yi})$, and yellow feature vector $\mathrm{My}=(\mathrm{Zi})$ respectively, $\mathrm{i}=$ $1,2,3$, for color difference threshold determination, in order to further confirm whether there is the rear brake light or not, determination methods are as follows:

If it meets the threshold conditions, i.e., $(|\mathrm{M} 1, \mathrm{Mr}|>|\mathrm{M} 2, \mathrm{Mr}|) \& \&(|\mathrm{M} 1, \mathrm{Mr}|-\mid$ $\mathrm{M} 2, \mathrm{Mr} \mid>=\mathrm{c})$ or $(|\mathrm{M} 1, \mathrm{Mw}|>|\mathrm{M} 2, \mathrm{Mw}|) \& \&(|\mathrm{M} 1, \mathrm{Mw}|-|\mathrm{M} 2, \mathrm{Mw}|>=\mathrm{c})$ or $(\mid \mathrm{M} 1$, My $>\mid$ M2, My $\mid) \& \&(\mid$ M1, My $|-|$ M2, My $\mid>=c), \mid$ M1, Mr $\mid$ represents the color difference of two vectors, it indicates that the frontal vehicle is braking, go to step 4.Where $\mathrm{c}$ is statistical error threshold of color difference.

\subsubsection{Red or Yellow Car Brake Lights Recognition}

\section{A. Brake Lights Candidate Region Detection}

By making corresponding subtraction in $\mathrm{R}$ channel, $\mathrm{G}$ channel, and $\mathrm{B}$ channel for Picture Pic1 and Picture Pic2 respectively, binarization and conducting and operation, we can finally get candidate region of brake lights.

As is shown in Fig.8, (a), (b) two frame images to be compared, (c), (d) the middle portion of the two frame images, (e) binarized image in the middle portion of the two frame images, (f) extraction of the rectangular area of the brake light. 


\section{B. Brake Lights Regional State Recognition}

By dilating or eroding brake lights candidate region obtained from step A, we can get the result (e) which is shown in Figure 8, By finding contours, we can obtain the coordinates of the center of gravity of contours. then we extract the feature vector of the $5 \times 5$ rectangular region (f) of Pic2, which is compared with a standard feature vector to make determination, the determination method is the same way as step B of step 3.2.2 where the two frames have small differences. If it meets the threshold conditions, it indicates that the frontal vehicle is braking, go to step 4 .
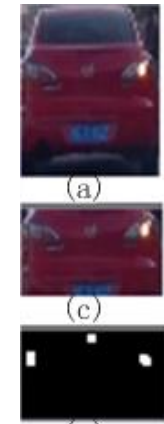

(e)
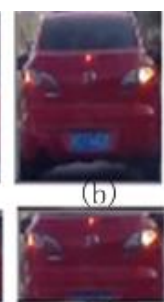

(d)

D

(f)

\section{Figure 8. Red or Yellow Car Brake Light Region Extraction Schematic Diagram}

\section{Outputs of Brake Lights Status}

If it detects inserting road warning, the third brake light or rear brake light, which indicating that the vehicle in front brakes or insert road, alerting the rear vehicle and it would make intelligent driving decisions automatically, slowing down or stoping by itself.

\section{Results and Discussion}

The Figure 9 is the comparison diagram of the small civilian cars' actual braking situations and the output of the system on sunny days. In Figure 9, comparison curves are represented in blue and green lines. The comparison results of the system output and actual braking situations are used as a reference to calculate the braking situations recognition rate. The experimental statistical results of recognition rate of different vehicle models in different kind of weather are shown in Table 1 and Table 2.

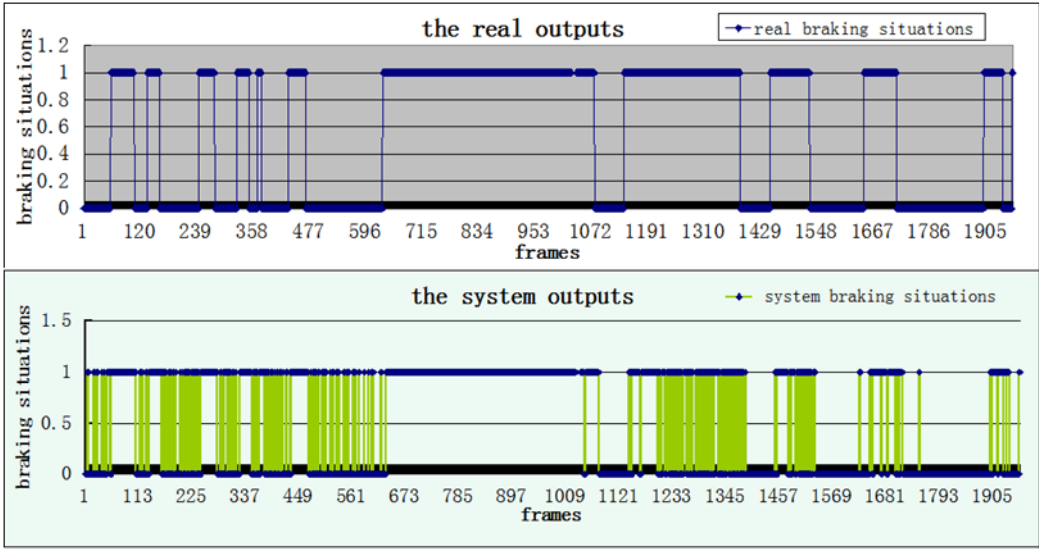

Figure 9. The Comparison of the System Outputs with the Real Braking Situations 
Table 1 shows recognition rate of the experiment which adopts the proposed method by using 4736 non-red and non-yellow colors cars' brake lights pictures in different situations detected from different videos. Table 2 shows recognition rate of the experiment which adopts the proposed method by using 635 red or yellow colors cars' brake lights pictures in different situations detected from different videos. Where RT is the false detection rate that brake lights are bright that is detected as not bright and FT is the false detection rate that brake lights are not bright that is detected as bright.

\section{Table 1. The Brake-light Recognition Accuracy Rate Evaluated for Different Vehicles in Different Weather}

\begin{tabular}{|c|c|c|c|c|c|c|c|c|}
\hline \multirow{2}{*}{$\begin{array}{c}\text { Types } \\
\text { of vehicle }\end{array}$} & \multicolumn{4}{|c|}{ Sunny } & \multicolumn{4}{|c|}{ Rainy } \\
\hline & $\begin{array}{c}\text { Number } \\
\text { of } \\
\text { images }\end{array}$ & RT & FT & $\begin{array}{c}\text { Recogn } \\
\text { ition } \\
\text { rate }\end{array}$ & $\begin{array}{c}\text { Number } \\
\text { of } \\
\text { Images }\end{array}$ & RT & FT & $\begin{array}{c}\text { Recogn } \\
\text { ition } \\
\text { rate }\end{array}$ \\
\hline Civilian cars & 1983 & $12.52 \%$ & $16.65 \%$ & $85.62 \%$ & 880 & $14.88 \%$ & $23.23 \%$ & $81.36 \%$ \\
\hline Buses & 523 & $12.15 \%$ & $20.01 \%$ & $84.32 \%$ & 430 & $15.61 \%$ & $24.35 \%$ & $80.47 \%$ \\
\hline Trucks & 520 & $13.29 \%$ & $22.22 \%$ & $82.69 \%$ & 400 & $15.45 \%$ & $25.56 \%$ & $80.01 \%$ \\
\hline
\end{tabular}
Table 2. The Brake-light Recognition Accuracy Rate Evaluated for Red
Small Car in Different Weather

\begin{tabular}{|c|c|c|c|c|c|c|c|c|}
\hline \multirow{2}{*}{$\begin{array}{c}\text { Types } \\
\text { of vehicle }\end{array}$} & \multicolumn{4}{|c|}{ Sunny } & \multicolumn{4}{|c|}{ Rainy } \\
\hline & $\begin{array}{c}\text { Number } \\
\text { of } \\
\text { images }\end{array}$ & $\mathbf{R T}$ & FT & $\begin{array}{c}\text { Recogn } \\
\text { ition } \\
\text { rate }\end{array}$ & $\begin{array}{c}\text { Number } \\
\text { of } \\
\text { images }\end{array}$ & $\mathbf{R T}$ & FT & $\begin{array}{c}\text { Recogn } \\
\text { ition } \\
\text { rate }\end{array}$ \\
\hline Civilian cars & 320 & $36.93 \%$ & $53.47 \%$ & $55.25 \%$ & 315 & $40.46 \%$ & $60.56 \%$ & $50.31 \%$ \\
\hline
\end{tabular}

From the Table 1, we can find that the recognition rate of frontal non-red and nonyellow colors cars' brake lights not only relates to the weather conditions (sunny day, rainy day), but also depends on the vehicle models. Specifically, we can find these three types of vehicles have higher recognition accuracy on sunny days than rainy days through horizontal comparison. By longitudinal comparison, the small civilian cars generally have higher recognition rate than buses and trucks in all weather conditions. Compared with the rear brake lights, the main reason is that the small civilian cars are fitted with the third brake light which are easier to identify the braking conditions of the front vehicle. On the 
other hand, the quality of braking light image is better on sunny days compared with that on rainy days.

The table 2 shows us that the recognition rate of front small red and yellow color car is higher on sunny days than on rainy days, but the overall recognition rate is not high. The main reason is that searching for the brake lights on a red color car is very hard.

\section{Conclusions}

By extracting color features, shape features and structural features from the brake lights area and making color difference threshold determination in the RGB color space to output the brake lights's status information of the frontal vehicle immediately and accurately, this can be applied to driver assistance and autopilot. The experiment shows that the recognition rate of brake lights of different vehicles whose color was not red or not yellow is more than $82 \%$ in the sunny days, and more than $80 \%$ in rainy days; the recognition rate was not high for the red or yellow vehicles on the whole, but it is better in sunny days than in rainy days; What's more, the proposed method in this paper is able to identify many kinds of vehicles brake lights, including different shapes and different locations. In addition, the calculating method of proposed method is faster than the normal, and the processing time of the adjacent frames is about $90 \mathrm{~ms}$ more or less. Experiments prove that it is of practicality.

In this study, the maximum vehicle speed is limited to $80 \mathrm{~km} / \mathrm{h}$ and there is enormous room for improvement. If the speed is faster than $80 \mathrm{~km} / \mathrm{h}$ in intelligent driving decisions, it needs to integrate radar and other sensors to process and the brake light recognition rate is not high, when the cars is red or yellow. Moreover, because of the influence of various light, all kinds of vehicles false detection rate should be further reduced in different bad weather. So it needs to do more research on identifying the brake lights of red or yellow cars, recognition rate of different vehicle models under different light, and integration of radar processing to improve recognition accuracy to perfect intelligent driving decisions.

\section{Acknowledgements}

This work was supported by the Project of Construction of Innovative Teams and Teacher Career Development for Universities and Colleges Under Beijing Municipality (CIT\&TCD20130513), the National Natural Science Foundation of China (Grant No.61271370), and the General Program of Science and Technology Development Project of Beijing Municipal Education Commission of China(SQKM201411417004).

\section{References}

[1] W. Y Wang, M. C. Lu, and C. Y Chu, "Nighttime Vehicle Distance Measuring System (NVDMS). IEEE Transactions on Circuits and System-JI, vol. 54, (2007) January.

[2] J. H. Park and C. S. Jeong, "Real-time Signal Light Detection", Proc. International Conf. on Future Generation Communication and Networking Symposia (2008).

[3] C. Yu, C. Huang, and Y. Lang, "Traffic Light Detection during Day and Night Conditions by a Camera", ICSP2010 Proceedings, (2010).

[4] M. Diaz-Cabrera1, P. Cerri2 and J. Sanchez-Medina, "Suspended Traffic Lights Detection and Distance Estimation Using Color Features", 15th International IEEE Conference on Intelligent Transportation Systems, (2012).

[5] G. Siogkas, E. Skodras and E. Dermatas, "Traffic Lights Detection in Adverse Conditions Using Color,Symmetry and Spatiotemporal Information", Proceedings of the International Conference on Computer Vision Theory and Applications,(2012).

[6] J. W. Gong, Y. H. Jiang, G. M. Xiong, C. H. Guan, G.Tao, and H. Y. Chen, "The Recognition and Tracking of Traffic Lights Based on Color Segmentation and CAMSHIFT for Intelligent Vehicles”, Proc. IEEE Intelligent Vehicles Symposium, (2010).

[7] C.-c. Wang, S.-S. Huang, L.-c. Fu, and P.-Y. Hsiao, "Driver Assistance System for Lane Detection and Vehicle Recognition with Night Vision", IEEE International Conference on Intelligent Robots and Systems, (2005). 
[8] S. Nagumo, H. Hasegawa, and N. Okamoto, "Extraction of Forward Vehicles by Front-mounted Camera Using Brightness Information", IEEE International Conference on Electrical and Computer Engineering, vol. 2, (2003) May, pp. 1243- 1246.

[9] N. Alt, C. Claus, and W. Stechcle, "Hardware/Software Architecture of an Algorithm for Vision-based Real-time Vehicle Detection in Dark Environments", In Proc. Design, Automation and Test in Europe, (2008) March, pp. 176 - 181.

[10] Y.-L. Chen, Y-H. Chen, C.-J. Chen, and B.-F. Wu, "Nighttime Vehicle Detection for Driver Assistance and Autonomous Vehicles", In Proc. IEEE 18th International Conference on Pattern Recognition, vol. I, (2006), August, pp. 687-690.

[11] R. O' Malley, E. Jones and M. Glavin, "Rear-lamp Vehicle Detection and Tracking in Low-Exposure Color Video for Night Conditions [J]", IEEE Trans on Intelligent Transportation Systems, vol. 11, no. 2, (2010), pp. 453-462.

[12] P. F. Alcantarilla, L. M. Bergasa and P. Jim'enez, "Night Time Vehicle Detection for Driving Assistance Lightbeam Controller [C]”, Proc of IEEE Symposium on Intelligent Vehicles, [S.1.]:IEEE Press, (2008), pp. 291-296.

[13] R. O' Malley, M. Glavi and E. Jones, "Vehicle Detection at Night Based on Tail-Light Detection", Proc. International Symposium on Vehicular Computing Systems, (2008).

[14] T. Schamm, C. von Carlowitz and J. Marius Z"ollner, "On-Road Vehicle Detection during Dusk and at Night”, Proc. IEEE Intelligent Vehicles Symposium, (2010).

[15] P. Thammakaroon and P. Tangamchit, "Predictive Brake Warning at Night Using Taillight Characteristic", Proc. IEEE, International Symposium on Industrial Electronics (2009).

[16] Y.-1. Chen, B.-f. Wu C.-j. Fan, "Real-time Vision-based Multiple Vehicle Detection and Tracking for and Night Time Traffic Surveillance [C], Proc of IEEE International Conference on Systems, Man and Cybernetics, (2009), pp. 3352-3358.

[17] Y.-Y. Lu, C.-C. Han and M.-Chih Lu, "Vision-based System for the Prevention of Car Collisions at Night", Machine Vision and Applications (2011)22:117127 DOI 10.1007/s00138-009-0239-2.

[18] R. A. Gupta and W. E. Snyder, "Detection of Multiple Preceding Cars in Busy Traffic Using Taillights", Image Analysis and Recognition 8th International Conference, ICIAR 2011, Proceedings, (2011).

[19] C. Fernandez and D. F. Llorca, "Real-time Vision-based Blind Spot Warning System: Experiments with Motorcycles in Daytime/Nighttime Conditions", International Journal of Automotive Technology, vol. 14, no. 1, (2013), pp. 113-122.

[20] O. Ryota and O. Gosuke, "Vehicles Detection Based on Extremas in Nighttime Driving Scene", 1st IEEE Global Conference on Consumer Electronics 2012, GCCE 2012, (2012), pp. 679-682.

[21] D.-Y. Chen and Y.-H. Lin, "Frequency-tuned Nighttime Brake-light Detection", In International Conference on Intelligent Information Hiding and Multimedia Signal Processing, (2010), pp. 619-622.

[22] D.-Y. Chen, Y.-H. Lin and H.-P. Kuo, "Scattering-based Brake-light Modeling and Detection", 14th International IEEE Conference on Intelligent Transportation Systems Washington, DC, USA. October 5-7, (2011).

[23] D.-Y. Chen, Y.-H. Lin, and Y.-J. Peng, "Nighttime Brake-Light Detection by Nakagami Imaging”, IEEE Trans on Intelligent Transportation Systems, vol. 13, no. 4, (2012) December.

[24] D.-Y. Chen and C.-H. Chen, "Salient Video Cube Guided Nighttime Vehicle Braking Event Detection", Journal of Visual Communication and Image Representation, vol. 23, no. 3, (2012) April, pp. 586-597.

[25] M. Casares, A. Almagambetov and S. Velipasalar, "A Robust Algorithm for the Detection of Vehicle Turn Signals and Brake Lights", IEEE Ninth International Conference on Advanced Video and SignalBased Surveillance, (2012).

[26] Y. Freund, R. E. Schapire , "Experiments with a New Boosting Algorithm [A]", In Proceedings of the 13th Conference on Machine Learning, Morgan Kaufmann [C], USA, (1996), pp. 148- 156.

[27] P. Viola and M. Jones, "Rapid Object Detection Using a Boosted Cascade of Simple Features [A]", In Proceeding of International Conference on Computer Vision and Pattern Recognition [C], Kauai, HI, USA (2001), vol. 1, pp. 511- 518.

[28] P. Viola and M. Jones, "Robust Real Time Face Detection [J]", International Journal of Computer Vision, Published by Springer, vol. 57, no. 2, (2004), pp. 137- 154.

[29] R. Lienhart and J. Maydt, "An Extended Set of Haar Like Features for Rapid Object Detection [A]", The IEEE International Conference on Image Processing [C],New York, USA, vol. 1, (2002), pp. 900-903. 


\section{Authors}
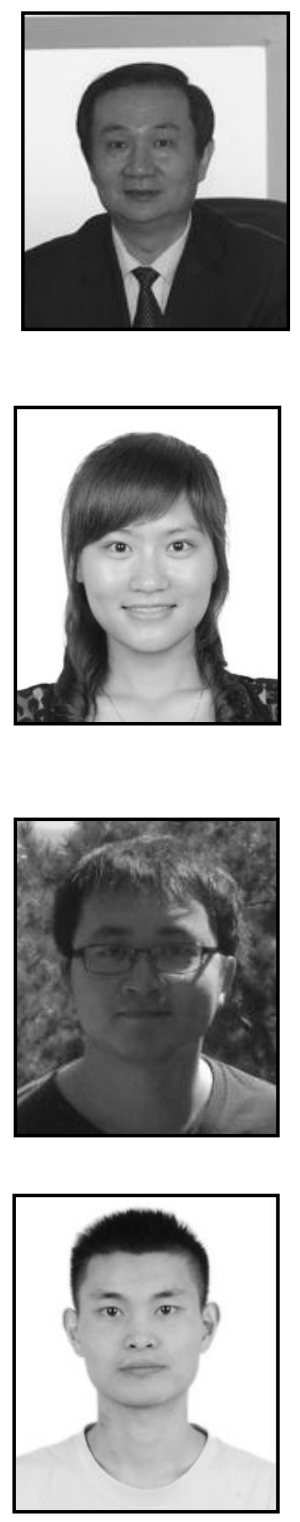

Hong Bao, corresponding author, male, he was born in Beijing, China, received his Ph.D. degree in computer science from school of computer and information technology from Beijing Jiao tong University Beijing, China and M.S. degree in information technology from Liverpool University, U.K. He is now a professor of Beijing Union University, Beijing, China. His current research interests include image processing, visual perception and distributed system.

Wei Liu, female, she was born in HuNan, China, Oct. 1989, received her Bachelor of Engineering degree in Communication Engineering from Beijing Union University, China. She is studying as a graduate student in Beijing Union University now. Her research interests concentrate on image processing.

Cheng Xu, male, was born in Inner Mongolia, China, Dec. 1988, received his Bachelor of Engineering degree in Communication Engineering from Beijing Union University, China. He is studying as a graduate student in Beijing Union University now. His research interests concentrate on image processing and visual perception.

Jun Zhang, male, he was born in ChongQing, China, Apr. 1988, received his Master of Science degree in Mathematics and Applied Mathematics from Chengdu University of Technology, China. He is working as a Algorithm Engineer in ChongQing now. His research interests concentrate on Remote sensed image processing. 
International Journal of Signal Processing, Image Processing and Pattern Recognition Vol.8, No.6 (2015) 